\title{
ON THE DISCRETE REPRESENTATIONS OF THE GENERAL LINEAR GROUPS OVER A FINITE FIELD
}

\author{
BY G. LUSZTIG
}

Communicated by Michael F. Atiyah, October 9, 1972

\begin{abstract}
In this note we present a construction for a distinguished representation in the discrete series of $G L_{n}(F), F$ a finite field. This is used in describing explicitly Brauer's lifting of the identity representation of $G L_{n}(F)$.
\end{abstract}

Let $V$ be a vector space of dimension $n \geqq 2$ over a finite field $F$ with $q$ elements. Let $W_{F}$ be the ring of Witt vectors associated to $F$ (see Serre $[4, I, \S 6])$ and $K_{F}$ its field of fractions. Denote by $x \rightarrow \bar{x}$ the canonical ring homomorphism $W_{F} \rightarrow F$ and by $y \rightarrow \tilde{y}$ the canonical multiplicative homomorphism $F^{*} \rightarrow W_{F}$ such that $\tilde{y}^{-}=y$ for $y \in F^{*}$.

Our purpose is to construct explicitly a free $W_{F}$-module $D(V)$ associated canonically to $V$, which regarded as a representation of $G L(V)$ belongs to the discrete series, i.e. its character is a cusp form on $G L(V)$. We could call $D(V)$ the distinguished representation of the discrete series of $G L(V)$.

The construction is as follows (the details will appear elsewhere). Let $X$ be the set of all sequences $\left(A_{0} \subset A_{1} \subset A_{2} \subset \cdots \subset A_{n-1}\right)$ of affine subspaces of $V\left(\operatorname{dim} A_{i}=i\right)$ which are away from the origin, i.e. $0 \notin A_{n-1}$. Let $\mathscr{F}$ be the set of all functions $f: X \rightarrow W_{F}$. Consider the subset $\mathscr{F}^{\prime} \subset \mathscr{F}$ consisting of all $f$ 's satisfying

(1) Given any fixed sequence $\left(A_{0} \subset A_{1} \subset \cdots \subset A_{i-1} \subset A_{i+1} \subset \cdots \subset\right.$ $\left.A_{n-1}\right)$ of affine subspaces of $V$ away from the origin and a variable $A_{i}$ between $A_{i-1}$ and $A_{i+1}$ away from the origin (there are $q$ choices for $A_{i}$ if $i=0, n-1$ and $q+1$ choices if $0<i<n-1)$, we have

$$
\sum_{A_{i}} f\left(A_{0} \subset A_{1} \subset \cdots \subset A_{i-1} \subset A_{i} \subset A_{i+1} \subset \cdots \subset A_{n-1}\right)=0 .
$$

Define $\mathscr{F}_{-1}^{\prime}$ as the set of all $f \in \mathscr{F}^{\prime}$ satisfying the homogeneity condition

(2) $f\left(\lambda A_{0} \subset \lambda A_{1} \subset \cdots \subset \lambda A_{n-1}\right)=\tilde{\lambda}^{-1} f\left(A_{0} \subset A_{1} \subset \cdots \subset A_{n-1}\right)$, $\forall \lambda \in F^{*}$.

It is clear that $\mathscr{F}, \mathscr{F}^{\prime}, \mathscr{F}_{1}^{\prime}$ are finitely generated free $W_{F}$-modules. Define a $W_{F}$-linear map $t: \mathscr{F} \rightarrow \mathscr{F}$ by the formula

(3) (tf $)\left(A_{0} \subset A_{1} \subset \cdots \subset A_{n-1}\right)=(-1)^{n-1} \sum f\left(A_{0}^{\prime} \subset A_{1}^{\prime} \subset \cdots \subset A_{n-1}^{\prime}\right)$, where the sum is extended over all $\left(A_{0}^{\prime} \subset A_{1}^{\prime} \subset \cdots \subset A_{n-1}^{\prime}\right)$ in $X$ such that $A_{0}^{\prime} \in A_{n-1}-A_{n-2}, A_{1}^{\prime}\left\|0 A_{0}, A_{2}^{\prime}\right\| 0 A_{1}, \ldots, A_{n-1}^{\prime} \| 0 A_{n-2}$ (observe that once $A_{0}^{\prime}$ is chosen, the $A_{i}^{\prime \prime}$ 's for $i>0$ are automatically determined so that

AMS (MOS) subject classifications (1970). Primary 20G40. 
the number of terms in the sum equals $\left.q^{n-1}-q^{n-2}\right) .^{1}$

One can show that $t$ conserves the conditions (1) and (2), i.e. $t\left(\mathscr{F}^{\prime}{ }_{-1}\right) \subset$ $\mathscr{F}_{-1}^{\prime}$.

Proposition 1. The map $t \otimes 1: \mathscr{F}_{-1}^{\prime} \otimes_{W_{F}} F \rightarrow \mathscr{F}_{-1}^{\prime} \otimes_{W_{F}} F$ is idempotent. All eigenvalues of $t: \mathscr{F}_{-1}^{\prime} \rightarrow \mathscr{F}_{-1}^{\prime}$ lie in $W_{F}$. There is precisely one eigenvalue $\lambda(V) \in W_{F}$ (repeated several times) such that $\overline{\lambda(V)}=1$.

Clearly $\lambda(V) \in W_{F}$ is an invariant of the vector space $V$.

Proposition 2.

$$
\lambda(V)=\sum_{y \in F^{\prime} ; \text { trace }_{F^{\prime} / F} y=1} \tilde{y}^{-1}
$$

where $F^{\prime}$ is an extension of degree $n$ of the field $F$. (The sum has $q^{n-1}$ terms which belong to $W_{F^{\prime}}$, but after summing, the result lies in the subring $W_{F} \subset$ $W_{F}$.)

The fact that $\overline{\lambda(V)}=1$ is contained in the following more general identity valid for integers $k$ such that $k \equiv 1(\bmod q-1)$ :

$$
\begin{aligned}
\sum_{y \in F^{\prime} ; \text { trace }_{F^{\prime} / F} y=1} y^{-k}=1 & & \text { if } k \equiv q^{i}\left(\bmod q^{n}-1\right) \text { for some } i, \\
=0 & & \text { otherwise. }
\end{aligned}
$$

EXAMPLE. In case $n=2, q=2$ we have $\lambda(V)=-1$. In case $n=2$, $q=3$ we have $\lambda(V)=\sqrt{-2}-1$ where $\sqrt{-2} \equiv-1(\bmod 3)$.

Definition. $D(V)=\left\{f \in \mathscr{F}_{-1}^{\prime} \mid t f=\lambda(V) f\right\}$.

If $\operatorname{dim} V=1$ define $D(V)=\left\{f: V-0 \rightarrow W_{F} \mid f(\lambda x)=\tilde{\lambda}^{-1} f(x), \lambda \in F^{*}\right.$, $x \in V-0\}$.

This is then a finitely generated free $W_{F}$-module which is a direct summand of $\mathscr{F}_{-1}^{\prime}$. The general linear group $G L(V)$ operates naturally in $D(V)$ so that $D(V)$ becomes a representation space for $G L(V)$.

Next we describe some simplicial complexes associated to $V$. Given any partially ordered set $S$ one can consider the simplicial complex whose $k$-simplices are precisely the totally ordered subsets of $S$ having $k+1$ elements.

EXAMPLES. (a) $S=$ set of all affine subspaces of $V$ away from the origin, ordered by inclusion. Let $A(V)$ be the corresponding simplicial complex.

(b) $S=$ set of all proper linear subspaces of $V$ which are transversal to a given proper linear subspace $V^{\prime} \subset V$, ordered by inclusion. Let $T\left(V, V^{\prime}\right)$ be the corresponding simplicial complex.

(c) $S=$ set of all affine subspaces of $V$ strictly contained in a given hyperplane $H$ in $V$ ( $H$ away from the origin), ordered by inclusion. Let $C(H)$ be the corresponding simplicial complex. Note that $C(H)$ is canoni-

\footnotetext{
${ }^{1}$ The symbol || denotes "is parallel to."
} 
cally isomorphic to $T\left(V, V^{\prime}\right)$ where $V^{\prime}$ is the unique hyperplane through the origin parallel to $H$.

(d) $S=$ set of all proper linear subspaces of $V$ ordered by inclusion. The corresponding symplicial complex is the well-known Tits complex $T(V)$ of $V$.

Proposition 3. Let $\tilde{H}$ denote reduced integral homology. (a) $\tilde{H}_{i}(A(V))=0$ for $i \neq 0, n-1, \tilde{H}_{n-1}(A(V))$ is free abelian of rank $q^{n(n+1) / 2}+$ lower powers of $q$.

(b) $\tilde{H}_{i}\left(T\left(V, V^{\prime}\right)\right)=0$ for $i \neq 0, l-1, \tilde{H}_{l-1}\left(T\left(V, V^{\prime}\right)\right)$ is free abelian of $\operatorname{rank}\left(q^{n-l}-1\right)\left(q^{n-l+1}-1\right) \cdots\left(q^{n-1}-1\right), l=\operatorname{dim} V^{\prime}$.

(c) $\widetilde{H}_{i}(C(H))=0$ for $i \neq 0, n-2, \widetilde{H}_{n-2}(C(H))$ is free abelian of rank $(q-1)\left(q^{2}-1\right) \cdots\left(q^{n-1}-1\right)$.

Note that (c) is a consequence of (b). It is easy to see that

$$
H_{n-1}(A(V)) \otimes_{Z} W_{F}=\mathscr{F} .
$$

Define a coefficient system (or sheaf) $\mathscr{G}$ over the Tits complex $T(V)$ as follows: To any simplex $\sigma=\left(V_{i_{0}} \subset V_{i_{1}} \subset \cdots \subset V_{i_{k}}\right)$ of $T(V)$ (i.e., a flag of linear subspaces of $V$ ) we associated the vector space $\mathscr{G}_{\sigma}=V_{i_{0}}$. If $\sigma^{\prime}=\left(V_{i_{0}} \subset \cdots \subset \hat{V}_{i_{h}} \subset \cdots \subset V_{i_{k}}\right)$ is a face of $\sigma$, we have a natural map $\varphi_{\sigma \sigma^{\prime}}: \mathscr{G}_{\sigma} \rightarrow \mathscr{G}_{\sigma^{\prime}}$ defined as the identity $V_{i_{0}} \rightarrow V_{i_{0}}$ in case $h>0$ or the natural inclusion $V_{i_{0}} \rightarrow V_{i_{1}}$ in case $h=0$. It is clear that the system $\left(\mathscr{G}_{0}, \varphi_{\sigma \sigma^{\prime}}\right)$ form a coefficient system over $T(V)$ with respect to which one can consider simplicial homology.

Proposition 4. Assume $n>2$. We have $H_{i}(T(V) ; \mathscr{G})=0$ for $i \neq 0$, $n-2, H_{0}(T(V) ; \mathscr{G}) \cong V, H_{n-2}(T(V) ; \mathscr{G})=F$-vector space of dimension $(q-1)\left(q^{2}-1\right) \cdots\left(q^{n-1}-1\right)$. If $n=2$, there is a natural surjective homomorphism $H_{0}(T(V) ; \mathscr{G}) \rightarrow V$ whose kernel has dimension $q-1$ and is canonically isomorphic to the space of homogeneous polynomials of degree $q-2$ on $V$, with values in $F$.

Put

$$
\begin{aligned}
\tilde{H}_{n-2}(T(V) ; \mathscr{G}) & =\mathrm{H}_{n-2}(T(V) ; \mathscr{G}) & & n>2, \\
& =\operatorname{ker}\left(H_{0}(T(V) ; \mathscr{G}) \rightarrow V\right), & & n=2 .
\end{aligned}
$$

Proposition 5. There is a canonical isomorphism $D(V) \otimes_{W_{F}} F \cong$ $\tilde{H}_{n-2}(T(V) ; \mathscr{G})(n \geqq 2)$. In particular $\operatorname{rank}_{W_{F}} D(V)=(q-1)\left(q^{2}-1\right) \cdots$ $\left(q^{n-1}-1\right)$.

Proposition 6. Choose an affine hyperplane $H \subset V$ away from the origin. There is a canonical isomorphism (depending on $H$ )

$$
D(V) \cong \tilde{H}_{n-2}\left(C(H) ; W_{F}\right) .
$$


Proposition 7. Choose a proper linear subspace $V^{\prime} \subset V, \operatorname{dim} V^{\prime}=l$. There is a canonical isomorphism (depending on $V^{\prime}$ )

$$
D(V) \otimes_{W_{F}} K_{F} \cong D\left(V / V^{\prime}\right) \otimes_{W_{F}} \tilde{H}_{l-1}\left(T\left(V, V^{\prime}\right) ; K_{F}\right) .
$$

Remarks. Proposition 5 shows that the $F$-reduction of $D(V)$ can be described homologically. One can prove that as soon as $n \geqq 3$ the modular representation $D(V) \otimes_{W_{F}} F$ of $G L(V)$ is not irreducible. It contains exactly 2 simple factors for $n=3$. Proposition 6 identifies the restriction of $D(V)$ to the affine subgroup (i.e., the subgroup of all $\alpha \in G L(V)$ such that $\alpha(H)=H$ ) with a representation space described homologically. The homological description of the restriction of $D(V)$ to the affine subgroup has been also obtained independently by L. Solomon (to appear). It follows from results of S. Gel'fand [1] that this restriction is absolutely irreducible. This implies that $D(V)$ is an absolutely irreducible $G L(V)$ module.

Proposition 7 describes the restriction of $D(V)$ to any maximal parabolic subgroup of $G L(V)$. It is quite likely that the isomorphism of Proposition 7 holds also with $K_{F}$ replaced by $W_{F}$ (this is the case for $l=1$ or $\left.n-1\right){ }^{2}$ Applying Proposition 6 repeatedly one can get a factorization into an iterated tensor product of the restriction of $D(V) \otimes_{W_{F}} K_{F}$ to any parabolic subgroup of $G L(V)$. For example the restriction to a Borel subgroup of $G L(V)$ is a tensor product of $n-1$ representations of the Borel subgroup of dimensions $q-1, q^{2}-1, \ldots, q^{n-1}-1$.

Proposition 8. Define $D^{(k)}(V)=\sum_{V^{\prime} \subset V ; 0 \in V^{\prime} ; \operatorname{dim} V^{\prime}=k} D\left(V^{\prime}\right)(1 \leqq k \leqq n)$. Then (a) There is a canonical exact sequence $0 \rightarrow D^{(n)}(V) \otimes_{W_{F}} F \rightarrow D^{(n-1)}(V) \otimes_{W_{F}} F \rightarrow \cdots \rightarrow D^{(1)}(V) \otimes_{W_{F}} F \rightarrow V \rightarrow 0$.

(b) Let $V_{1} \subset V$ be a proper linear subspace of $V$ and let $\mathscr{U}\left(V_{1}\right)=\{\alpha \in$ $G L(V): \alpha \mid V_{1}=$ identity, $\alpha \mid V / V_{1}=$ identity $\}$ be the unipotent radical of the maximal parabolic subgroup corresponding to $V_{1}$. Decompose

$$
D^{(k)}(V) \otimes_{W_{F}} K_{F}=D_{\mathrm{I}}^{(k)}(V) \oplus D_{\mathrm{II}}^{(k)}(V)
$$

where the first summand is the part on which $\mathscr{U}\left(V_{1}\right)$ acts as identity and the second summand is the part on which $\sum_{\alpha \in \mathcal{U}\left(V_{1}\right)} \alpha$ acts as zero. Then there is a canonical exact sequence (depending on $V_{1}$ )

Moreover $D_{I}^{(n)}(V)=0$.

$$
0 \rightarrow D_{\mathrm{II}}^{(n)}(V) \rightarrow D_{\mathrm{II}}^{(n-1)}(V) \rightarrow \cdots \rightarrow D_{\mathrm{II}}^{(1)}(V) \rightarrow 0 .
$$

(c) $D^{(k)}(V)$ are absolutely irreducible.

COROLlary. Let $\beta: G L(V) \rightarrow W_{F}$ be the character of the virtual representation $D^{(1)}(V)-D^{(2)}(V)+\cdots+(-1)^{n-1} D^{(n)}(V)$. Then for any $\alpha \in$

\footnotetext{
${ }^{2}$ ADDED IN PROOF. This has been proved to be true in general.
} 
$G L(V), \beta(\alpha)=\Sigma \tilde{\lambda}$ where the sum is over the $n$ eigenvalues $\lambda$ of $\alpha$.

REMARKS. 1. $\beta$ is the classical Brauer lifting of the identity representation of $G L(V)$. Of course, in order to check that $\beta$ was indeed a character, Brauer had to use his characterization of characters in terms of elementary subgroups, while here the virtual representation corresponding to $\beta$ is constructed explicitly.

2. The complete description of the irreducible (complex) characters of the general linear group over a finite field is due to J.A. Green [2]. However explicit realizations of the discrete series representations were known only for $S L_{2}$ (see Tanaka [5]).

ACKNOWLEDGEMENTS. It is my pleasure to thank $\mathbf{R}$. Carter for many stimulating conversations. I want to thank also $M$. Kervaire for his help in proving Proposition 2(i). My interest in understanding Brauer's lifting of modular characters has been aroused by Quillen's paper [3].

\section{REFERENCES}

1. S. Gel'fand, Representations of the full linear group over a finite field, Mat. Sb. 83 (125) (1970), 15-41 = Math. USSR Sb. 12 (1970), 13-40. MR 42 \#7797.

2. J. A. Green, The characters of the finite general linear groups, Trans. Amer. Math. Soc. 80 (1955), 402-447. MR 17, 345.

3. D. Quillen, The Adams conjecture, Topology 10 (1970), 67-80. MR 43 \#5525.

4. J. P. Serre, Corps locaux, Hermann, Paris, 1968.

5. S. Tanaka, Construction and classification of irreducible representations of special linear group of the second order over a finite field, Osaka J. Math. 4 (1967), 65-84. MR 36 \#2714.

Mathematics Institute, University of Warwick, Coventry, England. 